\title{
ENRICHMENT OF HEAVY ELEMENTS \\ IN CLUSTERS OF GALAXIES
}

\author{
YUHRI ISHIMARU \\ National Astronomical Observatory, \\ 2-21-1, Mitaka, Osawa, Tokyo 181, Japan. \\ email: ishimaru@th.nao.ac.jp
}

\begin{abstract}
We study the roles of type Ia (SNIa) and type II supernovae (SNII) in enrichment of the intra-cluster medium (ICM). Considering stellar $[\mathrm{Fe} / \mathrm{H}]$ of ellipticals, iron mass in ICM to luminosity ratios, and $[\alpha / \mathrm{Fe}]$ of ICM, we show that $~ 53 \%$ of iron in ICM comes from SNIa. While clusters keep all of ejecta from ellipticals, we obtain the observational evidence for that groups of galaxies lose parts of the gas by supernovae-driven winds. This 'group wind' scenario is confirmed by an 1D hydrodynamical model.
\end{abstract}

\section{Introduction}

Clusters of galaxies are surrounded by hot ICM containing a large amount of heavy elements. Since the iron mass in ICM to total luminosity ratios (FeML) appears to take the universal value $\sim 0.01-0.02 M_{\odot} / L_{\odot}$, the iron in ICM should come from ellipticals (Arnaud et al. 1992; Renzini et al. 1993). We study the origin of iron in clusters, considering the shares of iron between ICM and ellipticals. Previously, since the stellar iron abundance of ellipticals was believed as high as $\sim 2 Z_{\odot}$, the iron yield was regarded to be enough high to enrich the ICM mainly by SNII. Moreover, it was reported that ASCA observations gave high values of $[\alpha / \mathrm{Fe}]$ to ICM (Mushotzky et al. 1996). These estimates seemed to support SNII enriched ICM. However, if all of iron in ICM is supplied only by SNII and SNIa products remain in halos of ellipticals, it contradicts to the observed iron abundance of halos which is lower than the solar value (e.g., Awaki et al. 1994). Thus, we reconsider these observational evidences, and then examine the roles of SNIa and SNII in iron enrichment of ICM, by using a chemical evolution 


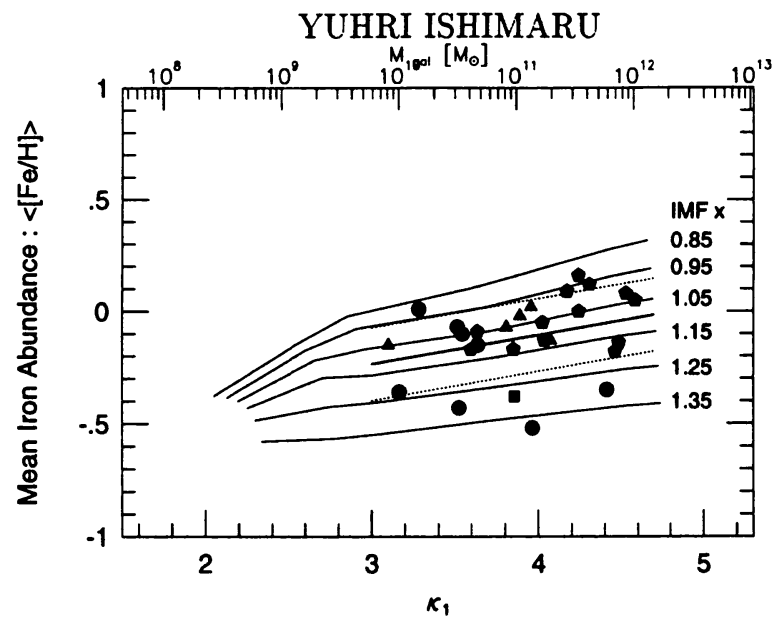

Figure 1. The mass-metallicity relation of ellipticals: The closed symbols indicate the observed relation based on the mean iron abundances. The tick solid and dotted lines indicate the regression line and the 1- $\sigma$ dispersions, respectively. The thin solid lines give the theoretical relations for the IMF $x=0.85,0.95,1.05,1.15,1.25$, and 1.35 . The value of $x$ is put at the next to the corresponding lines.

model based on a galactic wind scenario (cf. Arimoto \& Yoshii 1987). In addition, combining X-ray data for ellipticals and groups of galaxies as well as clusters, we examine the evolution of groups and discuss differences in evolutional histories due to the mass scales of galaxy systems.

\section{Clusters of Galaxies}

\subsection{MASS-METALLICITY RELATION OF ELLIPTICALS}

The previous values of high stellar abundances of ellipticals were derived from the central $\mathrm{Mg}_{2}$ index. However, since strong $\mathrm{Mg}_{2}$ index gradient exists in an elliptical, these values must be over-estimated. Therefore, taking into account abundance gradients, we have estimated mean stellar abundances for 50 ellipticals (Arimoto et al. 1996). The obtained mean values are as low as $\sim Z_{\odot}$. Thus, using the mass-metallicity relation given by the new data, we have examined the iron productivity of ellipticals. As clearly shown in Fig.1, the observed relation requires the IMF slope $x$ within a range of $1.10 \pm 0.1$, which is flatter than the Salpeter's IMF $(x=1.35)$.

\subsection{IRON MASS IN ICM TO TOTAL LUMINOSITY RATIOS}

The FeML indicates the efficiency of iron ejection from ellipticals. For the possible origins of iron in ICM, we consider i) SNII-driven galactic winds, ii) SNIa, and iii) stellar mass loss. We have calculated chemical evolutions of ellipticals with the various IMF slope; $x=0.85-1.35$, and the various binary frequency, which determines SNIa rate; $b / b_{\odot}=1 / 4-1$ (where, $b_{\odot}$ 

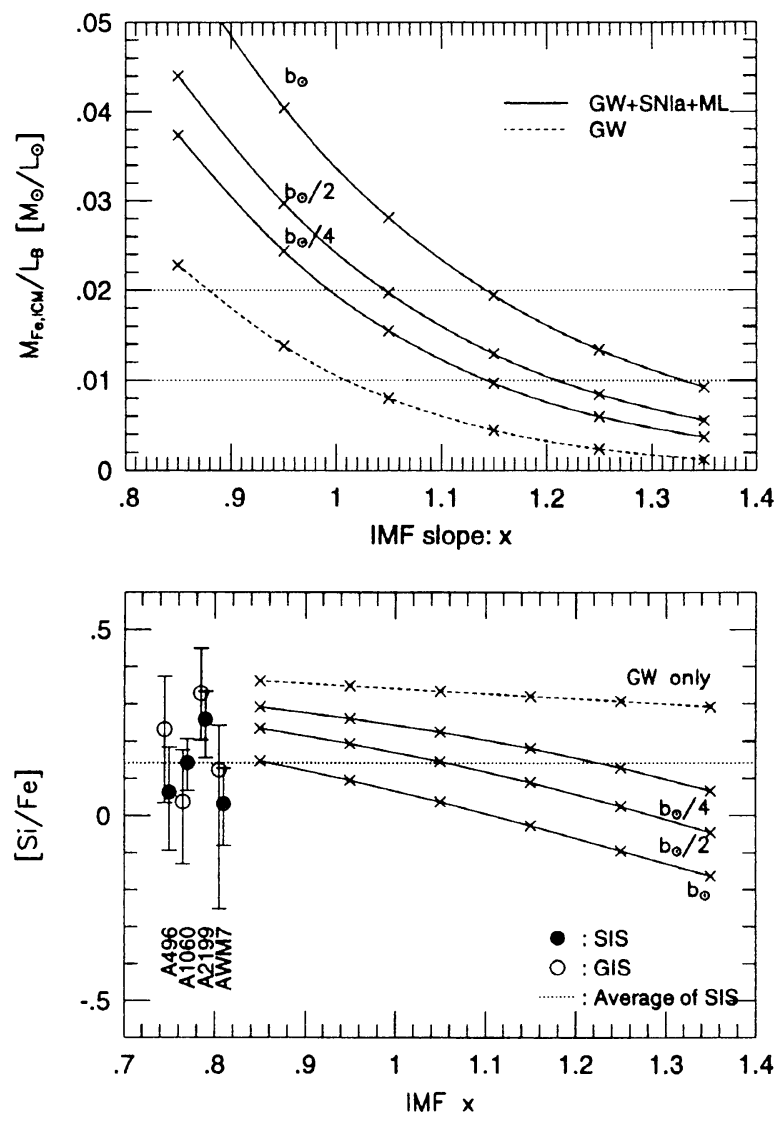

Figure 2. (upper) The relation between IMF slope $x$ and FeMLs: The dashed lines gives the case that the ICM is enriched only by galactic winds. The solid lines indicate the case that stellar mass loss and SNIa also contribute to the ICM enrichment, as well as galactic winds. The values of assumed binary frequency are put at the next to the corresponding lines. The dotted lines gives the range of observational IMLR distributions.

Figure 3. (lower) The relation between IMF slope $x$ and $[\alpha / \mathrm{Fe}]$ of ICM: The solid and dashed lines indicate the same way with Fig. 2. At the left side, the ASCA data renormalized by meteoritic solar abundances are given. The dotted lines indicate the mean value of the ASCA data.

is that for the solar neighbourhood). The predicted FeMLs are given by integrations of iron ejection from each ellipticals. As shown in Fig. 2, the observed range of FeML cannot be explained only by galactic winds, unless IMF slope is as flat as $x \sim 0.9$, which causes too high stellar abundances. But if the ICM was enriched by SNIa as well as SNII, the FeML and the mass-metallicity relation can be explained consistently with the combinations of $x$ and $b ;\left(1.15-1.20, b_{\odot}\right),\left(1.05-1.20, b_{\odot} / 2\right),\left(1.00-1.15, b_{\odot} / 4\right)$. 


\subsection{RELATIVE ABUNDANCE RATIOS OF ICM}

From recent ASCA observation, it has been reported that $[\alpha / \mathrm{Fe}]$ of ICM seem to take over-solar values (Mushotzky et al. 1996). However, this discussion is based on comparison with the solar-photospheric abundances. A serious discrepancy is known to exist in iron abundance between the solarphotospheric value and the meteoritic value (Anders \& Grevesse 1989). Thus, according to the prescription of supernovae nucleosynthesis by Nomoto et al., we have renormalized the ASCA data by the meteoritic values and examined the relative contributions of SNIa in ICM enrichment. As a result, the values of $[\alpha / \mathrm{Fe}]$ decrease by $\sim 0.16 \mathrm{dex}$ and the fractional contribution of SNIa in iron is more than 50\% (Ishimaru \& Arimoto 1997). As shown in Fig.3, clearly the observed $[\alpha / \mathrm{Fe}]$ requires SNIa products.

From these three observational constraints, we conclude that the $\sim 53 \%$ of iron in ICM comes from SNIa. In this case, ellipticals have a flat IMF slope $(x \sim 1.1)$ and a smaller binary frequency $b \sim b_{\odot} / 2$.

\section{Groups of Galaxies}

\subsection{ELLIPTICAL-GROUP-CLUSTER SEQUENCE}

Combining X-ray data of clusters, groups, and ellipticals, we have obtained the new aspects in evolutions of smaller scale galaxy systems. Figure 4 shows the relation between gravitational mass $M_{\text {grav }}$ and $M_{\text {gas }} / L_{B}$. There is a critical mass at $M_{\text {grav }} \sim 510^{13} M_{\odot}$, below which $M_{\text {gas }} / L_{B}$ is strongly correlated with $M_{\text {grav }}$. One can clearly identify the sequence from elliptical to clusters (hereafter EGC sequence). The EGC sequence is also established in the relation between $M_{\text {grav }}$ and FeML with the same critical mass. If this correlation is due to accretions of baryons, FeMLs must take the universal value. The decreases of gas and iron suggests that smaller groups lose more intra-group medium (IGM). Especially, the EGC sequences are smoothly connected to ellipticals. Thus, we can infer that small groups eject most of IGM by supernovae-driven winds like ellipticals. The efficiency of group winds should be negatively correlated with the binding energy, and therefore, the EGC sequence would emerge.

\subsection{GROUP WIND SCENARIO}

We examine that group winds can indeed reproduce the EGC sequence under the assumption of the universal initial baryon fraction, using the 1D hydrodynamical model. The evolution of IGM is examined for groups with $L_{B} \sim 510^{10}-510^{11} L_{\odot}$. The initial galaxy fraction is determined by the galaxy formation efficiency $\mathrm{GFE}=M_{\text {galaxy }} / M_{\text {baryon }}$. The observed value of $M / L_{V} ; \sim 70-150 M_{\odot} / L_{\odot}$ determines the possible range of GFE as $17 \pm 6 \%$. 

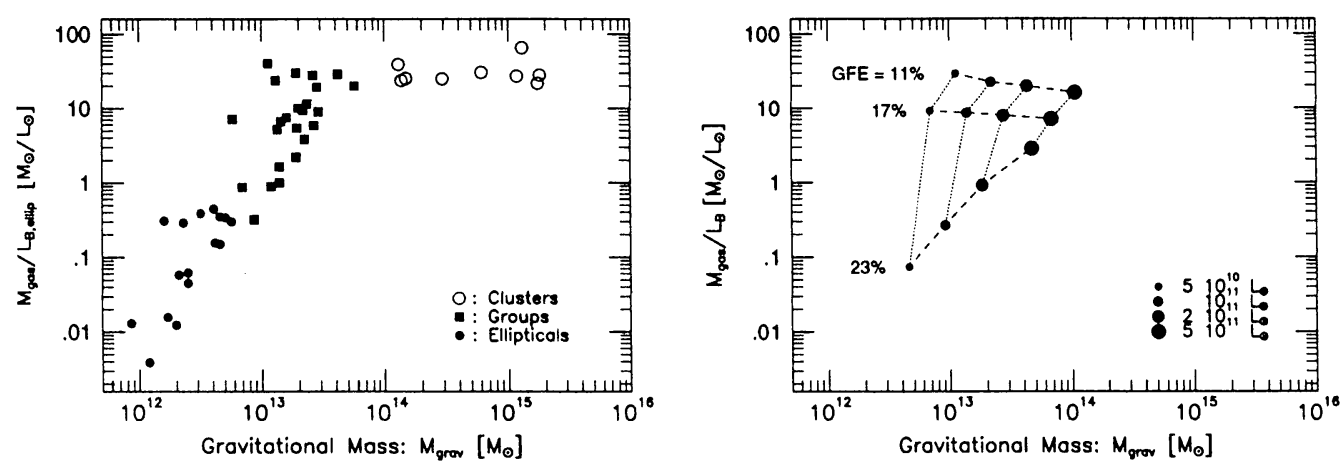

Figure 4. (left) The observed EGC sequence: The open circles, closed squares, and closed diamonds represent clusters, groups, and ellipticals, respectively.

Figure 5. (right) The calculated final gas-to-luminosity ratio as a function of gravitational mass: The total luminosities of each models are $510^{10}, 10^{11}, 210^{11}, 510^{11} L_{\odot}$, which are given by smaller to larger closed circles. The dotted and dashed lines connect models with the identical luminosities and galaxy formation efficiencies, respectively. The values of GFE are put at the side of corresponding lines.

Figure 5 shows the relation between the final $M_{\text {gas }} / L_{B}$ ratio and total mass. As shown in this figure, a small dispersion in GFE causes a large dispersion in the efficiency of gas ejection. Because the GFE represent the energy source in a unit gas mass, groups with higher GFE eject more IGM. In addition, smaller groups is more sensitive to the GFE. Therefore, a possible dispersion in GFE easily generate the observed trend and dispersion.

If rich clusters are formed through hierarchical clusterings, the EGC sequence requires that all group wind ejecta come back successfully at merging of groups. On the other hand, if each galaxy system has evolved isolately, the EGC sequence can be understood simply by group winds. Moreover, unnatural baryon enhancement in rich clusters is not required.

\section{References}

Anders, E., \& Grevesse, N. 1989, Geochim. Cosmochim. Acta, 53, 197

Arimoto, N., \& Yoshii, Y. 1987, A\&A, 173, 23

Arimoto, N., Matsushita, K., Ishimaru, Y., Ohashi, T., \& Renzini, A. 1997, ApJ, 477, 128

Arnaud, M., Rothenflug, R., Boulade, O., Vigroux, L., \& Vangioni-Flam, E. 1992, A\&A, 254,49

Awaki, H., Mushotzky, R. F., Tsuru, T., Fabian, A. C., Fukazawa, Y., Loewenstein, M., Makishima, K., Matsumoto, H., et al. 1994, PASJ, 46, L65

Ishimaru, Y., \& Arimoto, N. 1997, PASJ, 49, 1

Mushotzky, R., Loewenstein, M., Arnaud, K. A., Tamura, T., Fukazawa, Y., Matsushita, K., Kikuchi, K., \& Hatsukade, I. 1996, ApJ, 466, 686

Renzini, A., Ciotti, L., D'Ercole, A., \& Pellegrini, S. 1993, ApJ, 419, 52 